\title{
Hémorragies intra-alvéolaires
}

\author{
Pulmonary Alveolar Hemorrhage
}

\section{A. Parrot · G. Voiriot $\cdot$ A. Canellas $\cdot$ A. Gibelin $\cdot$ J.-M. Nacacche $\cdot$ J. Cadranel $\cdot$ M. Fartoukh}

Reçu le 19 avril 2018; accepté le 28 juin 2018

(C) SRLF et Lavoisier SAS 2018

Résumé L'hémorragie intra-alvéolaire (HIA), maladie rare, est une urgence thérapeutique, car elle peut conduire rapidement vers une insuffisance respiratoire aiguë asphyxiante avec décès. La triade, hémoptysie-anémie-infiltrat radiologique, suggère le diagnostic d'HIA, mais elle peut manquer dans deux tiers des cas, y compris chez des patients en détresse respiratoire. La tomodensitométrie thoracique peut aider dans les formes atypiques. Le diagnostic d'HIA repose sur la réalisation d'un lavage bronchoalvéolaire. Les étiologies en sont très nombreuses. Il importera de séparer, en urgence, les HIA d'origine non immune, avec un dépistage de celles d'origine septique qui doivent bénéficier d'une enquête microbiologique ciblée et cardiovasculaire avec la réalisation d'une échographie cardiaque, des HIA immunes (les vascularites liées aux anticorps anticytoplasme des polynucléaires neutrophiles, les connectivites et le syndrome de Goodpasture), avec la recherche d'autoanticorps et la réalisation de biopsies au niveau des organes facilement accessibles. La biopsie pulmonaire doit rester exceptionnelle. En cas d'HIA immune inaugurale, un traitement par stéroïdes et cyclophosphamide peut être débuté. Les indications du rituximab commencent à être mieux établies. Le bénéfice des échanges plasmatiques est débattu. En cas de réapparition d'infiltrats pulmonaires, chez un patient suivi pour une HIA immune, on s'efforcera d'écarter une infection dans un premier temps.

\footnotetext{
A. Parrot $(\bowtie) \cdot$ A. Canellas $\cdot$ J.-M. Nacacche $\cdot$ J. Cadranel

Service de pneumologie, pôle TVAR, hôpital Tenon,

hôpitaux universitaires de l'Est-Parisien, AP-HP,

4, rue de la Chine, F-75970 Paris cedex 20, France

e-mail : antoine.parrot@tnn.aphp.fr

G. Voiriot · A. Gibelin · M. Fartoukh

Service de réanimation et USC médicochirurgicale, pôle TVAR, hôpitaux universitaires de l'Est-Parisien, AP-HP, hôpital Tenon, F-75970 Paris cedex 20, France

G. Voiriot · A. Canellas · A. Gibelin · J. Cadranel · M. Fartoukh Sorbonne Université, Paris-VI, F-75005 Paris, France
}

Mots clés Hémorragie intra-alvéolaire - Vascularites · LEAD · Goodpasture · Infection · Insuffisance cardiaque

Abstract Diffuse alveolar hemorrhage (DAH), a rare disease, is a therapeutic emergency as it can lead quickly to asphyxiating acute respiratory failure (ARF) with death. The triad, hemoptysis-anemia-radiological infiltration, suggests the diagnosis of DAH, but it may be missed in two-thirds of cases, including the patients with respiratory distress. Thoracic computed tomography can help in atypical forms. The diagnosis of DAH is based on performing a bronchoalveolar lavage and the etiologies are numerous. It will be important to separate non-immune DAH with septicderived DAHs that should benefit from targeted microbiological investigation and cardiovascular disease by performing a cardiac ultrasound, from the immune DAHs (anticytoplasmic anti-neutrophil cytoplasmic vasculitis, connective tissue diseases and Goodpasture's syndrome) with research on autoantibodies and performing biopsies at easily accessible organs. Pulmonary biopsy must remain exceptional. In case of inaugural immune DHA, treatment with steroids and cyclophosphamide can be started. Indications for rituximab are beginning to be better established. The benefit of plasma exchange is debated. In case of recurrence of pulmonary infiltrates, in a patient followed for an immune DHA, we would strive to rule out an infection at first.

Keywords Diffuse alveolar hemorrhage - Vasculitis · LEAD $\cdot$ Goodpasture $\cdot$ Infection Heart failure

\section{Introduction}

L'hémorragie intra-alvéolaire (HIA), caractérisée par la présence de sang au niveau de l'acinus pulmonaire en rapport avec une lésion de la barrière alvéolocapillaire (excluant une inondation d'origine bronchique), est une maladie rare et sévère engageant le pronostic vital [1-3]. Entre 20 et $50 \%$ des patients sont ventilés et/ou dialysés [4,5]. La mortalité varie entre 20 et $100 \%$, avec une mortalité précoce attribuable 
à l'HIA d'au moins $10-30 \%$ [5-8]. De plus, chez certains patients, à l'atteinte pulmonaire est associée une atteinte rénale sévère pouvant conduire à la mise en dialyse chronique source d'une qualité de vie altérée [9]. Le pronostic des malades est largement amélioré par un diagnostic précoce, notamment dans les HIA d'origine immune par l'administration de corticoïdes et d'immunosuppresseurs, avec une mortalité inférieure à $20 \%$ [10-12]. Néanmoins, un tel traitement ne peut être banalisé, car il expose à un risque infectieux parfois mortel dans la moitié des cas, notamment lors de la phase d'induction du traitement $[12,13]$.

Poser le diagnostic est un défi, car les mécanismes conduisant à la lésion de la barrière alvéolocapillaire sont variés. Il peut s'agir d'une agression, mécanique secondaire à une élévation brusque de la pression veineuse capillaire (rétrécissement mitral [RM]), immunologique et/ou inflammatoire, avec la mise en évidence de dépôts de complexes immuns circulants (lupus érythémateux aigu disséminé [LEAD]), de dépôts membranaires alvéolaires d'immunoglobulines (syndrome de Goodpasture) ou de lésions de capillarite pulmonaire (vascularites).

La prise en charge de ces patients comprend l'identification de l'HIA, de sa cause et la mise en route du traitement. Cette mise au point n'abordera pas le patient immunodéprimé, suivi pour une maladie hématologique, ou transplanté [2].

\section{Diagnostic positif}

Typiquement, le diagnostic d'HIA est évoqué devant la triade associant hémoptysie, anémie et infiltrats pulmonaires radiologiques, avec un mode d'installation variable (insidieux à brutal). L'hémoptysie est rarement abondante du fait de son caractère distal. Au cours des HIA actives, l'anémie est toujours présente, parfois d'installation brutale, avec une perte brutale de 1 à $2 \mathrm{~g} / \mathrm{dl}$. La radiographie pulmonaire met en évidence des images micronodulaires à la phase initiale, qui ont bien souvent conflué vers des opacités alvéolaires à l'admission en réanimation. La tomodensitométrie (TDM) pulmonaire, non systématique d'autant qu'il peut exister une atteinte rénale, révèle des condensations, des opacités en verre dépoli et des réticulations pouvant donner un aspect de crazy paving.

En réalité, la triade classique, hémoptysie, anémie et infiltrats pulmonaires radiologiques, n'est présente que dans un tiers des cas [14]. Chacun de ces signes peut manquer. Le réanimateur peut être confronté à d'autres tableaux :

- un syndrome de détresse respiratoire aiguë sans hémoptysie, avec la constatation d'une chute rapide de l'hémoglobine sans diagnostic alternatif évident [15]. Le lavage bronchoalvéolaire (LBA) redressera le diagnostic en révélant un liquide uniformément rouge ;

- une hémoptysie sévère orientant vers une origine bronchique, mais à la radiographie, il y a des opacités alvéolaires bilatérales. L'angio-TDM peut aider dans ces situations difficiles en mettant en évidence une cause bronchique à l'hémoptysie, avec une hypervascularisation systémique ou une atteinte de la vascularisation pulmonaire ;

- des hémoptysies d'abondance variable associées à des opacités nodulaires multiples entourées de verre dépoli à la TDM, parfois associées à un épanchement pleural, qui font évoquer chez un malade non immunodéprimé un cancer métastatique (môle hydatiforme, cancer du testicule, angiosarcome...) (Fig. 1) [16].

Compte tenu de la diversité des présentations cliniques, le diagnostic d'HIA passe par la réalisation d'une endoscopie bronchique $[1,17]$. Elle élimine une cause locale de saignement et révèle en phase active des sécrétions sanglantes très aérées diffuses provenant des bronches sous-segmentaires. Le LBA confirmera le diagnostic avec trois aspects possibles en fonction de la chronologie par rapport au début du saignement et de son intensité [18]. En phase active, le liquide est uniformément hémorragique (deux tiers des patients dans notre expérience). En phase moins active, le liquide est rosé, avec de très nombreuses hématies en cytologie. Lorsque le LBA est fait plus à distance de l'épisode aigu, le liquide est clair ou grisé. C'est dans ce contexte que l'analyse cytologique prend son intérêt, pouvant retrouver encore des hématies, mais surtout plus de $20 \%$ de sidérophages ou un score de Golde (score quantifiant la charge en fer des macrophages grâce à la coloration de Perls qui colore ces derniers dans une gamme de bleu plus ou moins intense) supérieur à 20 (échelle allant de 0 à 400). Un score de Golde supérieur à 100 témoigne d'une HIA toujours importante. L'absence de sidérophages n'exclut pas le diagnostic en phase aiguë, les sidérophages apparaissant avec le temps (environ 72 heures). Quant à la biopsie pulmonaire, quelle que soit la méthode (transbronchique ou par vidéothoracoscopie), elle n'a pas d'indication pour confirmer le diagnostic.

\section{Diagnostic étiologique}

Les causes des HIA (Tableau 1) sont nombreuses. La classification idéale des HIA n'existe pas. Une classification sur les données histologiques est peu opérationnelle, car elle nécessite une biopsie qui n'est pratiquement jamais réalisée en période hémorragique, et la détection d'une lésion au sein d'une HIA active nécessite une expertise certaine. En l'état actuel des connaissances, nous séparerons les causes relevant d'un traitement par corticoïdes, voire d'autres immunosuppresseurs, de celles qui ne nécessitent pas de tels 


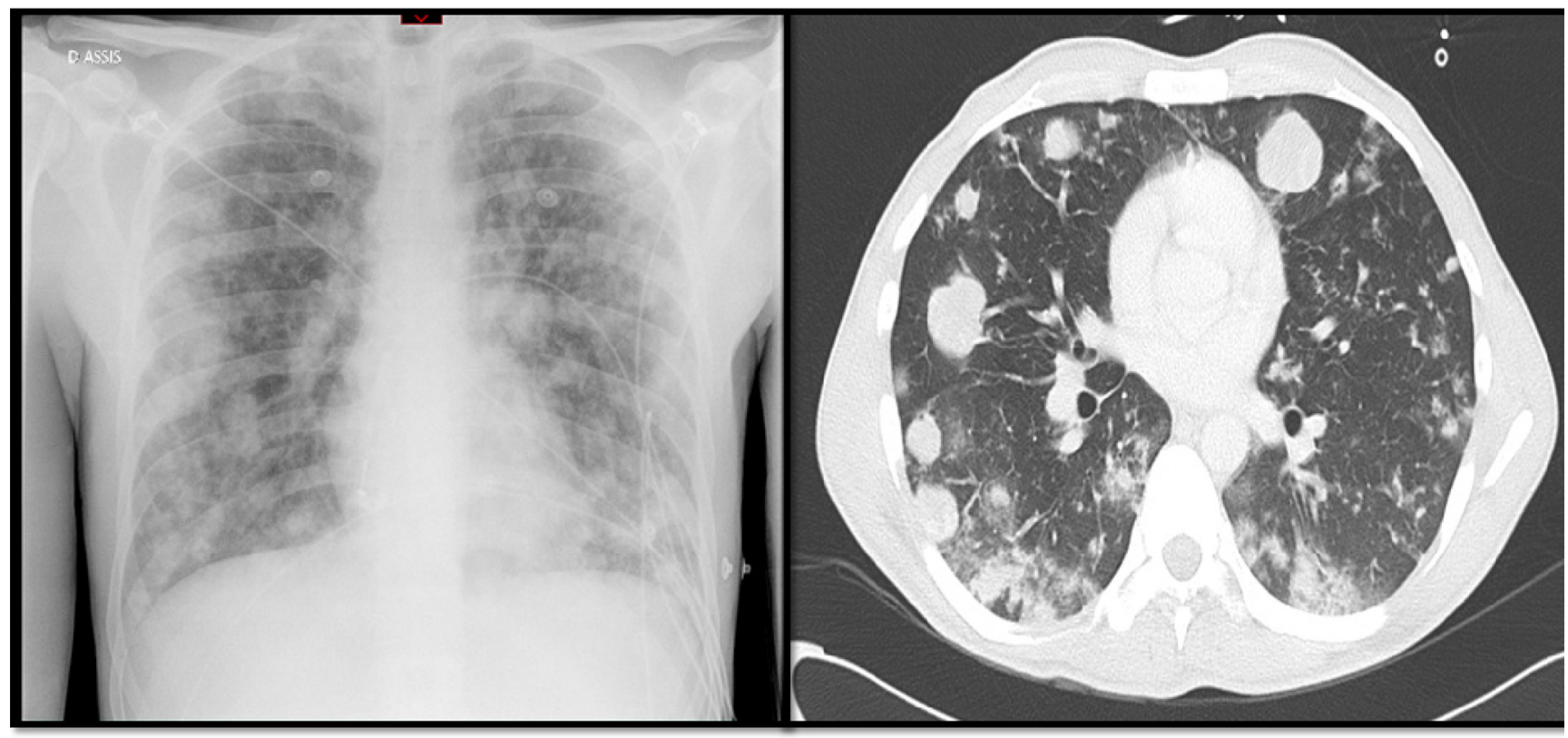

Fig. 1 HIA révélant un choriocarcinome ayant conduit à l'oxygénation extracorporelle du fait de l'intensité du saignement

traitements. Pour cette raison, les étiologies peuvent être distinguées en trois groupes :

- le premier groupe correspond aux HIA d'origine immune certaine ;

- le deuxième groupe comprend les HIA d'origine non immune ;

- le troisième groupe correspond aux HIA sans cause apparente (HIA idiopathiques).

Cette distinction peut paraître discutable, dans la mesure où certaines HIA non immunes sont possiblement liées à des mécanismes auto-immuns, telles que les HIA induites par des médicaments, par certains hydrocarbures ou par certaines infections telles que la leptospirose.

\section{HIA immunes}

\section{Vascularites}

Elles représentent la cause la plus fréquente des HIA immunes.

- Vascularites des petits vaisseaux

Vascularites à anticorps anticytoplasme des polynucléaires neutrophiles (ANCA)

Les vascularites à ANCA sont des maladies rares, l'incidence annuelle étant estimée entre 1 et 20 cas par million d'habitants, avec les plus fortes incidences pour la polyangéite microscopique (PAM) puis la granulomatose avec polyangéite (GPA ; anciennement maladie de Wegener) ; la granulomatose éosinophilique avec polyangéite (GEPA ; anciennement maladie de Churg et Strauss) est encore moins fréquente [19]. L'HIA, parfois inaugurale, survient essentiellement au cours des PAM et de la GPA dans 10 à $40 \%$ des cas, dont 20 à $50 \%$ sont des formes graves avec admission en réanimation $[4,12,20]$.

Polyangéite microscopique. La PAM est une vascularite nécrosante des petits vaisseaux. L'âge moyen des patients est de 50-60 ans sans prédominance de sexe [8]. À la différence $\mathrm{du}$ LEAD, l'atteinte pulmonaire est rarement brutale. Il existe le plus souvent une phase prodromique avec une altération de l'état général (trois quarts des cas). La PAM avec HIA réalise typiquement un syndrome pneumorénal, avec une hématurie présente dans plus de $90 \%$ des cas [8]. Les manifestations associées sont par ordre décroissant des manifestations rhumatologiques (arthralgies et myalgies : $52 \%$ ), cutanées (purpura vasculaire, ulcérations nécrosantes : $14 \%$ ), neurologiques (neuropathies multiples : $7 \%$ ) et oculaires (conjonctivite, épisclérite...). Les atteintes ORL (sinusite, épistaxis, surdité, ulcérations buccales) sont plus rares [8]. L'anémie est constante. Dans $90 \%$ des cas, il existe des ANCA, avec une fluorescence cytoplasmique périnucléaire (p-ANCA), dirigés contre la myéloperoxydase (MPO ; ANCA anti-MPO) dans plus de la moitié des cas ; dans un tiers des cas, la fluorescence est diffuse de type cytoplasmique c-ANCA dirigés contre des antiprotéinases 3 (PR3 ; ANCA anti-PR3) [8]. Une ponction-biopsie rénale (PBR) permettra de confirmer le diagnostic avec une glomérulonéphrite extracapillaire et évaluera le pronostic rénal [21]. Environ $5 \%$ des patients avec des p-ANCA ont de manière concomitante des anticorps anti-membrane basale glomérulaire (anti-MBG). Il paraît important de dépister 
Tableau 1 Étiologies des HIA (liste non exhaustive)

\section{HIA IMMUNES}

Vascularite des petits vaisseaux

Maladie à $A N C A$

Polyangéite microscopique

Granulomatose avec polyangéite

Granulomatose éosinophilique avec polyangéite

Capillarite pulmonaire pauci-immune

Vascularite à complexes immuns

Maladie avec anticorps anti-MBG

Vascularite cryoglobulinémique

Vascularite à IgA

Vascularite des vaisseaux de taille variable

Behçet

Vascularite liée à des maladies systémiques

LEAD

Polyarthrite rhumatoïde, sclérodermie, myopathies inflammatoires

Vascularite avec une étiologie probable

Hépatite $\mathrm{C}$ associée à une cryoglobulinémie

Vascularite médicamenteuse

Vascularite secondaire à un cancer

Syndrome des antiphospholipides

Autres causes

Maladie cœliaque

\section{HIA NON IMMUNES}

Pathologies cardiovasculaires

Rétrécissement mitral, myxome de l'oreillette

Insuffisance ventriculaire gauche

Maladie pulmonaire veino-occlusive

Troubles de l'hémostase

Médicaments/toxiques

Anticoagulants, fibrinolytiques

Propylthio-uracile, amiodarone

Cocaïne, crack

Infections

Grippe \pm Staphylocoque

Leptospirose

Dengue, hantavirus

Cancers

Choriocarcinome, môle hydatiforme

Angiosarcome

Métastases endovasculaires

Hémangioendothéliome épithélioïde, myélome

Edème à pression négative

Edème post-extubation

Convulsions

Autres

Embolie graisseuse, embolie de cholestérol

\section{HIA IDIOPATHIQUES}

HIA : hémorragie intra-alvéolaire ; anti-MBG : anticorps antimembrane basale glomérulaire ; LEAD ; lupus érythémateux disséminé

En gras : causes fréquentes ces patients, car le profil évolutif, au moins sur le plan rénal, est proche de celui des patients ayant un syndrome de Goodpasture, avec un bénéfice possible des échanges plasmatiques [22].

Granulomatose avec polyangéite. La GPA est une vascularite nécrosante atteignant les vaisseaux de petit et de moyen calibre, associée à des granulomes à cellules géantes, qui peut atteindre tous les organes. En cas d'HIA, la lésion pulmonaire prédominante est la capillarite pulmonaire, le granulome ou les microabcès rarement observés. L'âge moyen de survenue est de 50 ans, touchant plutôt des hommes. La GPA avec HIA s'accompagne le plus souvent d'une atteinte rénale et de la sphère ORL (rhinite, épistaxis, surdité, atteinte du cartilage) [23]. Cependant, l'hémoptysie peut être due à une atteinte bronchique par cette vascularite ou à une atteinte pulmonaire nécrosante [17]. Les autres atteintes (oculaires, neurologiques, cutanées) sont possibles mais plus rares. L'anémie est souvent présente. L'évolution peut être fulminante. Dans cette forme de GPA avec HIA, les ANCA sont présents dans $90 \%$ des cas, avec une fluorescence cytoplasmique (c-ANCA) et une spécificité de type ANCA antiPR3 [24]. Dans un peu moins de $10 \%$ des cas, il s'agit de pANCA. La PBR peut montrer une glomérulonéphrite extracapillaire associée à des lésions de vascularite et à des granulomes.

Granulomatose éosinophilique avec polyangéite. Au cours de cette maladie qui affecte des patients de 50 ans en moyenne, l'HIA est rare, avec une fréquence inférieure à $10 \%$ [25-27]. Sur un fond d'asthme, devenu parfois instable, d'une rhinite chronique et/ou une polypose nasale, d'une éosinophilie sanguine fluctuante, pouvant précéder l'HIA de plusieurs années, sont décrits deux phénotypes de la maladie. Le premier phénotype de type vascularite systémique des petits vaisseaux associe une HIA, une atteinte rénale (glomérulonéphrite extracapillaire avec des croissants), plus rarement une atteinte cutanée (purpura) et neurologique (mononévrites multiples) dans un contexte d'altération de l'état général avec myalgies, arthralgies et des ANCA positifs anti-MPO [25]. Le second phénotype est plutôt associé à une atteinte cardiaque avec une myocardite. Les ANCA sont négatifs. L'HIA est alors en rapport plutôt avec un œdème pulmonaire aigu hémorragique.

Capillarite pulmonaire pauci-immune. Elle est une entité rare (moins de 20 cas décrits), en partie sousestimée, car elle exige la réalisation d'une biopsie pulmonaire pour mettre en évidence des lésions de capillarite pulmonaire [28]. Elle affecte plutôt des sujets jeunes, de moins de 50 ans. À la phase prodromique, il existe souvent une atteinte des voies aériennes supérieures (rhinite, sinusite, otite). L'atteinte pulmonaire caractérisée par une HIA est isolée. Il n'y a en aucun cas d'atteinte rénale, cutanée, neurologique, cardiaque ou digestive. Elle est sévère, près de $40 \%$ des patients étant ventilés [28]. Le bilan immunologique, 
comprenant notamment la recherche d'ANCA, est le plus souvent négatif. Le pronostic est habituellement favorable avec un traitement associant corticoïdes et autres immunosuppresseurs. La récidive est exceptionnelle. Il n'est pas décrit de forme évoluant vers une atteinte multisystémique.

\section{Vascularite avec dépôts de complexes immuns}

Maladie des anticorps anti-membrane basale glomérulaire (syndrome de Goodpasture). Cette maladie rare avec une incidence de 0,5 cas par million d'habitants par an est caractérisée par la présence d'autoanticorps circulants dirigés contre des déterminants antigéniques conformationnels $\mathrm{du}$ collagène de type IV, qui se fixent sur les membranes basales glomérulaires et alvéolaires, sous la forme de dépôts linéaires et continus $[10,19]$. La mortalité approche $90 \%$ en l'absence de traitement adéquat, contrastant avec une survie à un an proche de $90 \%$ si elle est traitée [13]. La maladie de Goodpasture a une distribution bimodale en âge, avec un premier pic vers 20-30 ans et un second pic après 60 ans $[29,30]$. Il s'agit le plus souvent de patients fumeurs ou exposés à des produits chimiques ou à une infection virale. Souvent précédée d'une phase prodromique (fièvre à $38^{\circ} \mathrm{C}$, asthénie, amaigrissement), elle réalise un syndrome pneumorénal dans 50-80\% des cas [10,13]. L'hémoptysie est le symptôme inaugural le plus fréquent. L'atteinte rénale se traduit par une hématurie, une protéinurie ou une insuffisance rénale d'aggravation rapide. Chez les patients de plus de 65 ans, l'atteinte pulmonaire est moins fréquente, et l'atteinte rénale est moins sévère [31]. L'anémie est constante. De rares formes pulmonaires isolées sans atteinte rénale, parfois graves et relevant de l'assistance circulatoire extracorporelle (ECMO), sont décrites [29,32,33]. Le diagnostic repose sur la recherche d'anticorps anti-MBG circulants, par une technique fiable (RIA, Elisa), présents dans $90 \%$ des cas $[2,34]$; la recherche par simple immunofluorescence indirecte est rapide, mais peu sensible. La négativité des tests peut être due au fait que tous les anticorps sont fixés sur leur cible (poumon et rein) ou qu'il s'agit d'anticorps non détectés par les tests (qui détectent surtout la sous-classe IgG1) de type IgG4 ou IgA [10,35]. Ces " formes séronégatives » pourraient être plus fréquentes dans les formes pulmonaires isolées et/ou avec une atteinte rénale minime [35]. En cas de négativité, même si l'atteinte rénale paraît minime, la PBR permet de mettre en évidence une glomérulonéphrite extracapillaire nécrosante avec en immunofluorescence des dépôts linéaires d'immunoglobulines caractéristiques, le long des membranes basales glomérulaires. Près de $30 \%$ des patients avec anticorps anti-MBG présentent des ANCA positifs majoritairement de type anti-MPO. Ces patients « double positifs » ont un phénotype mixte : ils partagent avec les patients avec ANCA l'âge de 60 ans, la durée d'installation des symptômes, une meilleure survie rénale, le risque de récidive ; par contre, la fréquence d'atteinte pulmonaire est comparable à celle des patients avec des anticorps
anti-MBG circulants $[22,31,36]$. La survie de ces patients à un an est de près de $90 \%$ identique à celle des patients avec une maladie à ANCA ou avec une maladie de Goodpasture [36].

Vascularite à IgA (anciennement purpura rhumatoïde de Henoch-Schönlein). Au cours de la vascularite à IgA, l'atteinte pulmonaire est rare $(<5 \%)$, mais lorsqu'elle est présente, elle est dominée par une HIA [37]. Elle survient le plus souvent chez des patients dont la maladie est connue, avec une atteinte rénale, une glomérulonéphrite extracapillaire, avec dépôts granuleux d'IgA ( $90 \%$ des cas), digestive (94\% des cas), cutanée ( $86 \%$ des cas) et articulaire (73\% des cas). Dans les rares cas où la biopsie pulmonaire est effectuée, elle retrouve des dépôts d'IgA dans les parois alvéolaires [37]. La moitié des patients sont ventilés, et près de $30 \%$ décèdent.

- Vascularites pouvant atteindre les vaisseaux de taille variable et les gros vaisseaux

Au cours de la maladie de Behçet et de la maladie de Takayasu, l'hémoptysie liée à une capillarite pulmonaire est une cause exceptionnelle d'HIA. Il faudra évoquer avant tout une hémoptysie liée aux gros vaisseaux (anévrismes, embolies, sténoses).

- Vascularites liées aux maladies systémiques

Lupus érythémateux disséminé

Parmi les connectivites, le LEAD est la principale cause d'HIA. C'est une manifestation rare, avec une fréquence inférieure à $5 \%$, survenant le plus souvent au cours d'une maladie active [38]. En l'absence de traitement adapté, la mortalité est de l'ordre de 75 contre $30 \%$ à l'ère du traitement par corticoïdes-cyclophosphamide [39]. Elle atteint plutôt les femmes jeunes. L'installation sur le plan respiratoire est habituellement brutale, en moins de trois jours, deux tiers des patients étant ventilés [5]. Dans deux tiers des cas, l'hémoptysie est présente. Dans un tiers des cas, il existe une note pleurale. Dans près de trois quarts des cas, il existe un syndrome pneumorénal. L'anémie est quasi constante. Les autres manifestations extrapulmonaires (cutanées [alopécie, ulcérations buccales], neurologiques, hématologiques [leucopénie, anémie hémolytique, thrombopénie] ou rhumatologiques) sont d'autant plus rares ou moins intenses qu'il s'agit de patients traités par immunosuppresseurs. Biologiquement, il existe très souvent une hypocomplémentémie et des facteurs antinucléaires de type anti-ADN natif et/ou anti-Smith. La biopsie pulmonaire est peu utile.

Au cours d'une maladie traitée, l'HIA est souvent associée à une infection évolutive dans plus d'un tiers des cas, et il faudra éliminer un syndrome catastrophique des antiphospholipides dont l'une des composantes peut être une HIA et dont 
la prise en charge peut possiblement déboucher sur un traitement par anticoagulants [38].

\section{Autres connectivites}

De rares cas d'HIA ont été décrits au cours de la polyarthrite rhumatoïde, de la sclérodermie, parfois associés à une note de vascularite avec des ANCA positifs et des polymyosites ; mais dans ces cas, plutôt que d'évoquer une poussée de la maladie, il vaut mieux évoquer une cause non immune.

- Vascularites probablement secondaires

Elles peuvent être secondaires à un cancer, à un médicament ou à une infection (virus des hépatites $\mathrm{B}$ ou $\mathrm{C}$ ).

$\mathrm{Au}$ cours des vascularites secondaires à une cryoglobulinémie qualifiée de menaçante, due au virus de l'hépatite $C$, l'HIA est décrite dans un peu plus de $7 \%$ des cas. Elle en constitue un facteur de mortalité majeur, avec $80 \%$ de décès [40]. L'atteinte pulmonaire est associée à une atteinte rénale, cutanée, articulaire respectivement dans 72,55 et $16 \%$ des cas. La fièvre n'est présente que dans $28 \%$ des cas. Les taux de $\mathrm{C} 3$ et de $\mathrm{C} 4$ sont abaissés dans $90 \%$ des cas. La PBR retrouve une glomérulonéphrite membranoproliférative, des dépôts granuleux d'immunoglobulines et de complément, associés à des thrombus intraluminaux.

Certains traitements (hydralazine, D-pénicillamine, allopurinol et surtout propylthio-uracile) sont inducteurs de vascularites avec une capillarite pulmonaire et/ou la mise en évidence d'ANCA.

\section{Syndrome des antiphospholipides}

L'HIA, manifestation rare, du syndrome des antiphospholipides ( $2 \%$ des patients) est rarement inaugurale du diagnostic [41]. Elle survient dans deux cadres. Le premier est en rapport avec des thromboses multiples principalement microcirculatoires pouvant toucher de très nombreux organes conduisant au syndrome catastrophique des antiphospholipides avec une défaillance multiviscérale : atteinte rénale (dans $75 \%$ des cas) avec HTA, atteinte cardiaque avec insuffisance cardiaque, atteinte cutanée (livedo), atteinte neurologique et atteinte hématologique (thrombopénie [50\%] et anémie hémolytique [30\%]) avec des schizocytes [42]. Le second cadre est en rapport avec une note plus inflammatoire de la maladie caractérisée par une capillarite pulmonaire sans thrombose [43]. Elle affecte plutôt des hommes d'un âge médian de 43 ans et s'associe uniquement aux marqueurs biologiques de la maladie sans aucun antécédent d'événement thrombotique. Dans les deux cadres sont retrouvés à des titres élevés un anticoagulant circulant lupique, des anticorps anticardiolipines de type IgG (à un moindre degré des IgM) ou des anticorps anti- $\beta 2$-glycoprotéines 1 . La mortalité est élevée de l'ordre de $30 \%$ principalement en rapport avec une HIA non contrôlée [43].
Il importe de cerner ces deux cadres, car en cas de capillarite pulmonaire, on recommande plutôt d'arrêter ou de ne pas initier les anticoagulants, de traiter au moins par de fortes doses de corticoïdes, alors que l'on plaiderait plutôt pour les anticoagulants en cas de syndrome catastrophique $[41,43]$.

\section{HIA non immunes}

\section{HIA d'origine cardiovasculaire}

Une HIA peut compliquer une hypertension veineuse pulmonaire secondaire à un RM, dont le diagnostic n'est pas aisé avec un risque de mort subite, ou une dysfonction ventriculaire gauche sévère, caractérisée par une amélioration partielle avec des diurétiques et une résolution radiologique lente et parfois favorisée par un traitement anticoagulant [7]. Pour cette raison, nous recommandons de réaliser systématiquement une échographie cardiaque dans le bilan d'une HIA. L'HIA s'intègre parfois dans un tableau d'hypertension artérielle maligne qui, quand elle est associée à un syndrome pneumorénal, en impose pour une vascularite.

\section{HIA d'origine médicamenteuse et toxique}

Les principaux agents responsables sont répertoriés dans le site Pneumotox (http://www.pneumotox.com). Les médicaments les plus souvent incriminés sont utilisés en cardiologie : l'amiodarone, les anticoagulants classiques ou oraux directs, les antiagrégants, les fibrinolytiques [44,45]. L'inhalation de certains toxiques, comme la cocaïne et le crack ou le cannabis par la technique du " bang », entraîne la survenue d'HIA [46,47]. Les silicones employées à visée esthétique peuvent s'accompagner d'HIA. À la limite de cette revue, certaines chimiothérapies (gemcitabine), des anticorps monoclonaux et des immunosuppresseurs (sirolimus) sont sources d'HIA [17].

\section{HIA sur un oedème à pression négative}

Le tableau est évoqué sur les circonstances de survenue (en postopératoire chez des sujets plutôt jeunes ou après des épisodes convulsifs) [48].

\section{HIA d'origine septique}

Elles sont à repérer compte tenu de l'urgence à mettre en route le traitement approprié [3]. Elles sont dominées en Europe par certaines viroses comme la grippe maligne compliquée ou non d'une surinfection à Staphylococcus aureus producteur de la toxine de Panton-Valentine [49], dans les territoires d'outre-mer par la leptospirose [50,51], en 
Amérique par les hantaviroses et dans les tropiques par la dengue [3]. À l'heure des voyages, ces maladies surviennent en dehors des zones d'endémie.

Ces HIA septiques sont caractérisées par une première phase pseudogrippale plus ou moins intense après un délai d'incubation variable suivant l'agent responsable (quelques jours pour la grippe, entre deux jours et un mois pour la leptospirose, et de quatre à dix jours pour la dengue). Ensuite, la deuxième phase varie selon le germe en cause. Pour la leptospirose est observée une atteinte hépatique se traduisant par un ictère, une atteinte rénale et un syndrome hémorragique (épistaxis, pétéchies, hémorragie sousconjonctivale) $[50,52]$. Au cours de cette phase peut survenir l'atteinte pulmonaire qui peut être sévère et d'apparition très brutale avec une HIA. Parfois, l'atteinte pulmonaire survient en dehors de toute atteinte hépatique. Le diagnostic repose sur des hémocultures précoces (première semaine), avec un examen direct possible mais peu fiable et une culture positive peu utile, car elle est longue et nécessite des milieux spéciaux, une PCR qui se négative après dix jours, un Elisa qui détecte les IgM ou la technique de référence de microagglutination qui permettra de définir le sérotype. La mortalité peut dépasser $50 \%$. Pour la dengue à la défervescence thermique est décrit un syndrome de fuite capillaire plus ou moins intense (hypotension, épanchements des séreuses, hémoconcentration), un syndrome abdominal et des signes d'hémorragies avec rarement une HIA. Le diagnostic reposera sur des techniques de PCR, d'Elisa (détection de l'Ag NS1 spécifique de la dengue) pour une détection précoce ou les sérologies classiques pour une détection tardive.

\section{HIA d'origine tumorale}

Elles sont dominées par les choriocarcinomes [53], les sarcomes [16] et plus rarement par les hémangioendothéliomes. À l'exception des choriocarcinomes dont le diagnostic est évoqué devant un taux plasmatique de $\beta H C G$ très élevé, leur diagnostic nécessite un prélèvement pulmonaire histologique chirurgical.

\section{Autres causes}

De multiples autres causes à l'origine d'HIA sont inventoriées : embolies de cholestérol (le contexte de geste endovasculaire chez un patient athéromateux doit y faire penser), après un tremblement de terre... [17,18,54].

\section{HIA idiopathiques}

Elles constituent un groupe hétérogène, allant de l'hémosidérose idiopathique [55], en passant par des formes frustes de maladie systémique, à des HIA liées à des causes envi- ronnementales (exposition à certaines moisissures domestiques telles que Stachybotrys chartarum). Pour certaines, il existe probablement un mécanisme immunologique à l'origine de l'HIA. Le diagnostic est largement tributaire des investigations effectuées.

\section{Démarche diagnostique}

\section{Première situation : patient indemne d'affection responsable d'HIA}

La démarche repose :

- sur la répartition des causes d'HIA, qui dépend de la zone géographique et des examens réalisés. En France métropolitaine, les HIA sont d'origine immune dans $35 \%$ des cas, dominées par les vascularites à ANCA (PAM, GPA), d'origine cardiaque dans $29 \%$ des cas et de causes multiples dans $23 \%$ des cas ; elles étaient classées idiopathiques dans $13 \%$ des cas [14]. À l'île de La Réunion, la première cause d'HIA est la leptospirose ;

- une véritable enquête exhaustive (Tableau 2) $[14,17,24]$;

- des examens complémentaires parfois complexes. Le bilan biologique est centré sur la fonction rénale, les troubles de la coagulation et la recherche d'autoanticorps particuliers (Tableau 3). Il doit être étendu aux recherches infectieuses en cas de fièvre associée. L'échographie cardiaque est systématique pour l'évaluation de la fonction du ventricule gauche et la détection d'un rétrécissement mitral, car l'auscultation peut être faussement rassurante, $\mathrm{y}$ compris dans une forme très serrée. En cas de possible HIA immune, même si les examens immunologiques facilitent le diagnostic, la confirmation par une biopsie ciblée par les données précédentes d'un des organes atteints est parfois nécessaire [21]. Ainsi, la PBR, comprenant une étude histologique et une immunofluorescence, permet le diagnostic d'une glomérulonéphrite extracapillaire pauci-immune (vascularite à ANCA), à dépôts linéaires évocateurs de syndrome de Goodpasture et à dépôts granuleux (LEAD, néphropathie à $\operatorname{IgA}$, cryoglobulinémie). L'indication de la biopsie pulmonaire à visée étiologique, si possible par vidéothoracoscopie, doit être réservée aux HIA avec lésions extrathoraciques absentes ou non biopsiables et sans stigmate immunologique sérique : en particulier, si l'on redoute une infection ou une tumeur (par exemple un angiosarcome) ou si l'on envisage un traitement par immunosuppresseur tel que du cyclophosphamide dans le cadre d'une capillarite pulmonaire isolée [2]. Elle exige la collaboration d'un anatomopathologiste expert capable par exemple de poser le diagnostic de capillarite pulmonaire et d'angiosarcome ; 
Tableau 2 Enquête clinique d'une HIA

\begin{tabular}{|lll|}
\hline & & HIA cause \\
\hline Circonstances & Salle de réveil & Edèmes à pression négative \\
de survenue & Convulsions & Edèmes à pression négative \\
Mode d'installation & Accident de la voie publique avec fractures des os longs & Eraisseuse \\
Contexte & Installation brutale & LEAD, infection \\
& Période hivernale grippale & Grippe \\
& Baignade en eau douce & Leptospirose \\
Médicaments & Séjour tropical & Dengue \\
Prodromes & Anticoagulants, propylthio-uracile & Pneumopathies médicamenteuses \\
Signes cliniques & Syndrome grippal & Infections \\
& Rénaux (hématurie, protéinurie, insuffisance rénale rapidement & Vascularites \\
& progressive, HTA) & \\
& Rhumatologiques (arthralgies, arthrites, myalgies) & \\
& ORL (rhinite, perforation nasale, sinusite, épistaxis, surdité) & \\
& Oculaires (conjonctivite, épisclérite, etc.) & \\
& Cutanés (purpura, nodules sous-cutanés, livedo, ulcère digital) & \\
& Neurologiques (mono/multinévrite) & \\
& État de choc & Infections
\end{tabular}

Tableau 3 Bilan paraclinique

Bilan initial Bilan ciblé

NFS, plaquettes, TP, TCA

Schizocytes, haptoglobine, bilirubine, LDH

Créatininémie, urée, protéinurie/créatinurie, bandelette urinaire,

ECBU, cylindres hématiques urinaires

ECG, BNP, échographie cardiaque

Hémocultures

Examen direct et culture des prélèvements respiratoires

Autres

ANCA, ANTI-MBG (Elisa)

C3, C4, CH50

Anticorps antinucléaires \pm anti-ADN natifs

Sérologie des hépatites $\mathrm{B}$ et $\mathrm{C}$

Anticorps antiphospholipides, anticoagulant circulant lupique,

Anti- $\beta 2$-glycoprotéine 1

Autres

Biopsies
RT-PCR grippe

PCR et sérologie leptospirose

PCR et sérologie dengue

Facteur rhumatoïde, anticorps anticitruline

Cryoglobulinémie
Anticorps antigliadine, endomysium et antitransglutaminase ${ }^{a}$ $\beta \mathrm{HCG}$

Biopsie rénale avec immunofluorescence

Biopsies guidées par la clinique (neuromusculaire, ORL, peau, digestive $^{\mathrm{a}}$ )

${ }^{\text {a }}$ Maladie coliaque

- l'identification de certaines situations permettant de cibler les examens en fonction des données recueillies (Fig. 2) :

- les HIA d'origine septique dont nous avons décrit précédemment le tableau clinique justifient de faire pratiquer des hémocultures, des PCR (grippe, leptospirose, dengue, hantavirus, etc.) et les sérologies correspondantes ;
- les HIA avec des nodules doivent faire évoquer rapidement un choriocarcinome (un simple dosage de $\beta \mathrm{HCG}$ permet le diagnostic) ou un angiosarcome où il faudra discuter sans attendre la biopsie pulmonaire ;

- les HIA d'origine immune qui sont associées dans 60 à $80 \%$ des cas à un syndrome pneumorénal 


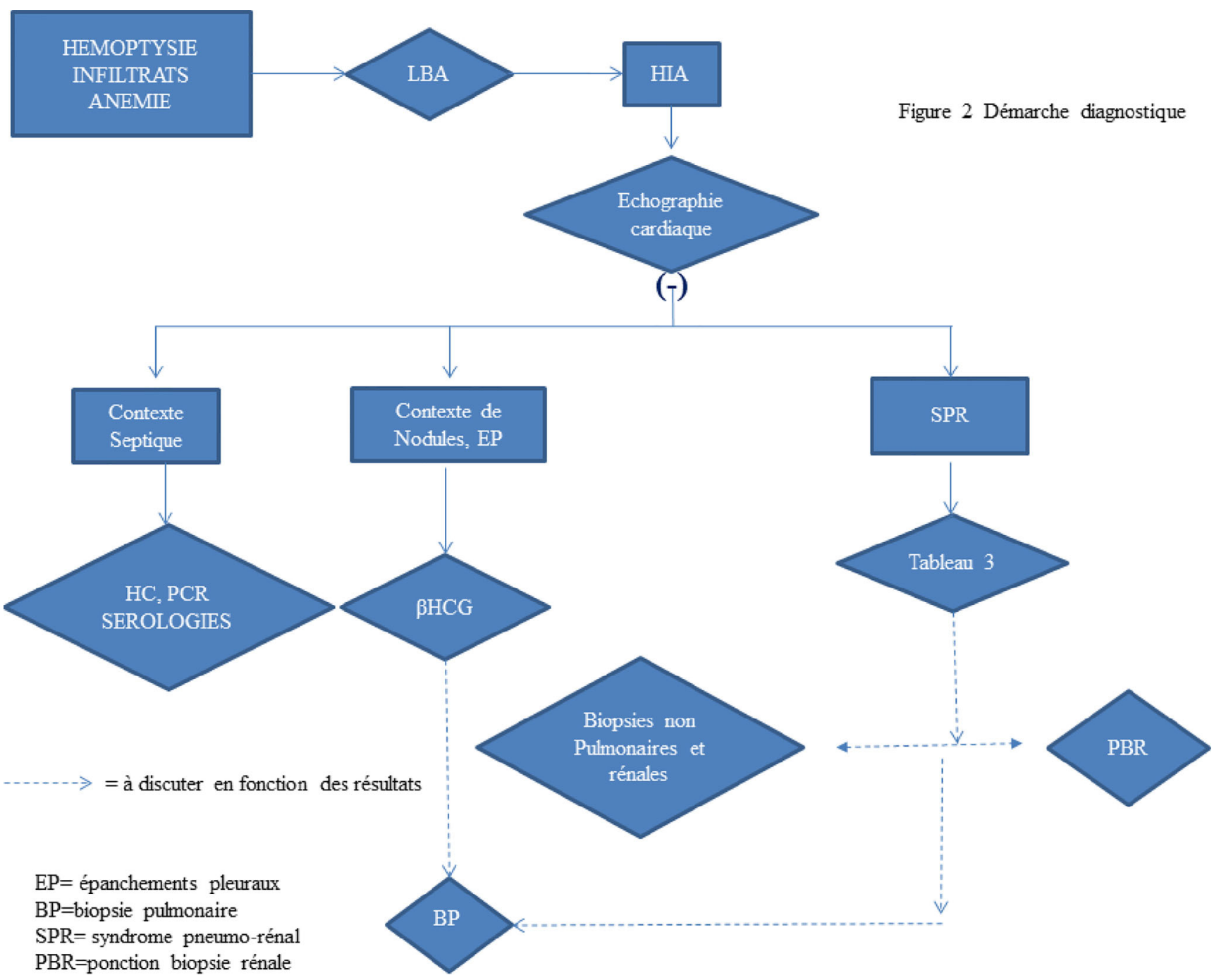

Fig. 2 Démarche diagnostique

(protéinurie ou hématurie ou insuffisance rénale rapidement progressive) évoluant dans un contexte d'altération de l'état général avec des signes respiratoires remontant à plusieurs jours (exception faite du LEAD où le tableau respiratoire peut être extrêmement brutal) [14]. Le bilan immunologique doit être exhaustif en s'efforçant d'obtenir en urgence notamment les dosages d'ANCA et d'anticorps anti-MBG. Néanmoins, la réalisation de certains examens tels qu'une biopsie est parfois difficile pour des raisons de gravité liées à l'état du patient ou des raisons techniques liées à la disponibilité des examens, il faudra alors essayer de porter le diagnostic d'HIA immune sur une combinaison d'éléments cliniques et biologiques. On peut s'aider du score clinicobiologique que nous avons développé et validé sur deux cohortes consécutives et qui peut se calculer au premier jour d'admission en réanimation (Tableau 4) $[5,56]$.
Seconde situation : patient connu pour une maladie réputée source d'HIA (maladie immunologique essentiellement)

Un traitement immunosuppresseur est souvent en cours. Par conséquent, avant de conclure à une poussée de la maladie, il faut s'acharner à écarter une infection, une surcharge et des effets iatrogènes (surdosage en anticoagulants par exemple). La stratégie diagnostique privilégie une enquête microbiologique exhaustive.

\section{Traitement}

\section{Mesures générales}

Le traitement symptomatique de l'insuffisance respiratoire aiguë n'a pas de spécificité et peut dans les formes les plus 


\begin{tabular}{|ll|}
\hline $\begin{array}{l}\text { Tableau } 4 \text { Score diagnostique en faveur d'une HIA } \\
\text { immune [56] }\end{array}$ \\
\hline Délai des symptômes respiratoires $\geq 11$ jours & 2 points \\
Asthénie, perte de poids dans le mois & 2 points \\
Arthralgies ou arthrites & 3 points \\
Protéinurie $\geq 1 \mathrm{gr} / 1$ & 3 points \\
\hline Un score supérieur ou égal à 4 est en faveur d'une HIA d'ori- \\
gine immune
\end{tabular}

graves recourir à l'oxygénation extracorporelle qui peut être initiée sans anticoagulation du circuit. Une antibiothérapie est souvent débutée au cours des HIA d'origine septique. La volémie doit être appréciée régulièrement, toute surcharge étant délétère, notamment en cas d'insuffisance rénale associée. Toute anomalie de l'hémostase peut aggraver la maladie : les traitements antiagrégants ou anticoagulants non indispensables doivent être interrompus. Certains proposent, notamment dans les formes très menaçantes d'HIA immunes, du facteur VII activé (par voie intraveineuse, plus rarement par lavage ou nébulisation pour diminuer le risque de complications thrombotiques) [57].

\section{HIA non immunes}

Le traitement étiologique des formes non immunes dépend de la cause. Il peut se résumer à l'arrêt du facteur déclenchant (médicament, barotraumatisme). En cas de rétrécissement mitral, il est urgent de se mettre en contact avec un service de chirurgie cardiaque. Au cours de la leptospirose, le traitement repose sur la pénicilline ou les céphalosporines de troisième génération. Dans les formes graves, certains ont recours à la desmopressine ou à la corticothérapie dont l'efficacité est controversée $[51,58]$.

\section{HIA immunes}

Les objectifs du traitement d'induction sont les suivants :

- contrôle rapide de l'HIA ;

- prévention de l'évolution vers des dégâts irréversibles tels qu'une insuffisance rénale chronique ;

- contrôle de la maladie causale en évitant un surtraitement source de complications infectieuses et tumorales.

En urgence, seule la corticothérapie permettra le contrôle rapide de l'HIA et limitera l'évolution vers des dégâts irréversibles. Le traitement d'attaque est fondé sur une corticothérapie à forte dose, administrée en bolus $(15 \mathrm{mg} / \mathrm{kg}$ par exemple sans dépasser $1 \mathrm{~g}$, trois jours de suite), avec un relais à la dose de $1 \mathrm{mg} / \mathrm{kg}$ au moins durant la première semaine [38]. À cette corticothérapie il est indispensable d'associer un immunosuppresseur qui est actuellement le plus souvent du cyclophosphamide dont l'action salvatrice n'est pas immédiate. Le schéma habituellement recommandé pour le cyclophosphamide est le suivant : une administration en bolus de $600 \mathrm{mg} / \mathrm{m}^{2}$ à j0, j14, j28 puis $700 \mathrm{mg} / \mathrm{m}^{2}$ toutes les trois semaines (sans dépasser $1200 \mathrm{mg}$ ) pour trois à six bolus supplémentaires, avec un total maximal de neuf bolus, en adaptant les posologies à l'âge et à la fonction rénale [59]. La voie intraveineuse est moins toxique que la voie orale. Ce schéma thérapeutique bien codifié dans les maladies à ANCA est utilisé dans le LEAD ou le syndrome de Goodpasture [60] ou dans les autres formes graves de vascularites rares [1], à l'exception des cryoglobulinémies graves. À la place du cyclophosphamide pourrait se discuter le rituximab qui a montré dans les vascularites à ANCA sa non-infériorité dans des formes non graves [61]. En 2013, les recommandations du Groupe français d'étude des vascularites étaient de ne pas proposer le rituximab en première intention chez les patients de réanimation (patient ventilé ou créatinémie $>350 \mu \mathrm{mol} / \mathrm{l}$ ), dans le syndrome de Goodpasture ou la GEPA [62]. En 2016, le groupe EULAR (European League Against Rheumatism) n'exclut pas son recours en première intention, notamment chez les patients en âge de procréer, car le cyclophosphamide est source de stérilité [63].

Si le recours aux échanges plasmatiques paraît cohérent avec la physiopathologie, en épurant notamment les anticorps circulants sources des lésions, leur utilisation est débattue. Dans la maladie de Goodpasture, même si les niveaux de preuve sur des études robustes manquent, les échanges plasmatiques associés au cyclophosphamide et aux corticoïdes restent le traitement de référence. Ils améliorent la survie rénale et globale des patients, d'autant plus que le patient n'est pas d'emblée dialysé [64,65]. Jusqu'à preuve du contraire, en cas d'HIA, même si la récupération d'une fonction rénale est compromise (créatinine $>600 \mu \mathrm{mol} / \mathrm{l}$ ), les échanges plasmatiques doivent être proposés. Dans les vascularites, une méta-analyse récente suggérait un bénéfice des échanges plasmatiques au cours des vascularites sur le pronostic rénal mais pas sur la survie [66], mais les premiers résultats de l'étude randomisée PEXIVA montrent que les échanges plasmatiques n'apportent aucun bénéfice sur un index composite le décès ou l'insuffisance rénale terminale. Dans le LEAD réfractaire et la cryoglobulinémie, l'indication reste à établir. En pratique, au cours du syndrome de Goodpasture, les échanges plasmatiques sont quotidiens durant 14 jours au minimum, jusqu'à négativation du titre des anticorps anti-MBG, à la dose de $60 \mathrm{ml} / \mathrm{kg}$, avec un maximum de 4 1/jour [10]. Au cours des vascularites à ANCA, la dose est de $60 \mathrm{ml} / \mathrm{kg}$, avec un minimum de sept échanges sur 14 jours [21]. Le cyclophosphamide ou le rituximab doivent être administrés après la première séance. Les échanges plasmatiques doivent utiliser des plasmas frais congelés au cours de ces formes hémorragiques. 


\section{Conclusion}

La prise en charge thérapeutique de l'HIA est urgente, car elle peut évoluer vers le décès. Les causes sont multiples. Il importera de séparer les HIA d'origine non immune, septique et cardiovasculaire, avec la réalisation d'une échographie cardiaque des HIA immunes avec la recherche d'autoanticorps et la réalisation de biopsies au niveau des organes facilement accessibles. La biopsie pulmonaire doit rester exceptionnelle. En cas d'HIA immune inaugurale, un traitement par stéroïdes peut être débuté. Par contre, chez un patient suivi pour une maladie de système susceptible d'engendrer une HIA, on s'efforcera d'écarter une infection dans un premier temps.

Liens d'intérêts : les auteurs déclarent ne pas avoir de lien d'intérêt.

\section{Références}

1. Casian A, Jayne D, (2011) Management of alveolar hemorrhage in lung vasculitides. Semin Respir Crit Care Med 32: 335-345

2. Lara AR, Schwarz MI, (2010) Diffuse alveolar hemorrhage. Chest 137: 1164-1171

3. von Ranke FM, Zanetti G, Hochhegger B, Marchiori E, (2013) Infectious diseases causing diffuse alveolar hemorrhage in immunocompetent patients: a state-of-the-art review. Lung 191: 9-18

4. Cartin-Ceba R, Diaz-Caballero L, Al-Qadi MO, Tryfon S, Fervenza FC, Ytterberg SR, Specks U, (2016) Diffuse alveolar hemorrhage secondary to antineutrophil cytoplasmic antibodyassociated vasculitis: predictors of respiratory failure and clinical outcomes. Arthritis Rheumatol 68: 1467-1476

5. Quadrelli S, Dubinsky D, Solis M, Yucra D, Hernandez M, Karlen H, Brigante A, (2017) Immune diffuse alveolar hemorrhage: clinical presentation and outcome. Respir Med 129: 59-62

6. Benoit FL, Rulon DB, Theil GB, Doolan PD, Watten RH, (1964) Goodpasture's syndrome: a clinicopathologic entity. Am J Med 37: 424-444

7. de Prost N, Parrot A, Picard C, Ancel PY, Mayaud C, Fartoukh M, Cadranel J, (2010) Diffuse alveolar hemorrhage: factors associated with in-hospital and long-term mortality. Eur Respir J 35: 1303-1311

8. Lauque D, Cadranel J, Lazor R, Pourrat J, Ronco P, Guillevin L, Cordier JF, (2000) Microscopic polyangiitis with alveolar hemorrhage. A study of 29 cases and review of the literature. Groupe d'études et de recherche sur les maladies « orphelines » pulmonaires (GERM"O"P). Medicine 79: 222-233

9. Li ZY, Gou SJ, Chen M, Zhao MH, (2013) Predictors for outcomes in patients with severe ANCA-associated glomerulonephritis who were dialysis-dependent at presentation: a study of 89 cases in a single Chinese center. Semin Arthritis Rheum 42: 515-521

10. Henderson SR, Salama AD, (2018) Diagnostic and management challenges in goodpasture's (anti-glomerular basement membrane) disease. Nephrol Dial Transplant 33: 196-202

11. Kimmoun A, Baux E, Das V, Terzi N, Talec P, Asfar P, Ehrmann S, Geri G, Grange S, Anguel N, Demoule A, Moreau AS, Azoulay E, Quenot JP, Boisrame-Helms J, Louis G, Sonneville R, Girerd N, Ducrocq N, Agrinier N, Wahl D, Puechal X, Levy B, (2016) Outcomes of patients admitted to intensive care units for acute manifestation of small-vessel vasculitis: a multicenter, retrospective study. Crit Care 20: 27

12. Solans-Laque R, Fraile G, Rodriguez-Carballeira M, Caminal L, Castillo MJ, Martinez-Valle F, Saez L, Rios JJ, Solanich X, Oristrell J, Pasquau F, Fonseca E, Zamora M, Callejas JL, Frutos B, Abdilla M, Fanlo P, Garcia-Sanchez I, Lopez-Dupla M, Sopena B, Perez-Iglesias A, Bosch JA; Spanish Registry of systemic vasculitis from the Autoimmune Diseases Study Group of the Spanish Society of Internal Medicine, (2017) Clinical characteristics and outcome of Spanish patients with ANCA-associated vasculitides: impact of the vasculitis type, ANCA specificity, and treatment on mortality and morbidity. Medicine 96: e6083

13. Huart A, Josse AG, Chauveau D, Korach JM, Heshmati F, Bauvin E, Cointault O, Kamar N, Ribes D, Pourrat J, Faguer S; French Society of Hemapheresis, (2016) Outcomes of patients with Goodpasture syndrome: a nationwide cohort-based study from the French Society of Hemapheresis. J Autoimmun 73: 24-29

14. de Prost N, Parrot A, Cuquemelle E, Picard C, Antoine M, Fleury-Feith J, Mayaud C, Boffa JJ, Fartoukh M, Cadranel J, (2012) Diffuse alveolar hemorrhage in immunocompetent patients: etiologies and prognosis revisited. Respir Med 106: 1021-1032

15. Rabe C, Appenrodt B, Hoff C, Ewig S, Klehr HU, Sauerbruch T, Nickenig G, Tasci S, (2010) Severe respiratory failure due to diffuse alveolar hemorrhage: clinical characteristics and outcome of intensive care. J Crit Care 25: 230-235

16. Chen YB, Guo LC, Yang L, Feng W, Zhang XQ, Ling CH, Ji C, Huang JA, (2010) Angiosarcoma of the lung: 2 cases report and literature reviewed. Lung cancer 70: 352-356

17. Traclet J, Lazor R, Cordier JF, Cottin V, (2013) Alveolar hemorrhage. Rev Med Interne 34: 214-223

18. Parrot A, Fartoukh M, Cadranel J, (2015) Alveolar hemorrhage. Rev Mal Respir 32: 394-412

19. Gibelin A, Maldini C, Mahr A, (2011) Epidemiology and etiology of wegener granulomatosis, microscopic polyangiitis, churg-strauss syndrome and goodpasture syndrome: vasculitides with frequent lung involvement. Semin Respir Crit Care Med 32: 264-273

20. Hirayama K, Kobayashi M, Usui J, Arimura Y, Sugiyama H, Nitta K, Muso E, Wada T, Matsuo S, Yamagata K ; Japanese RSGoPRD, (2015) Pulmonary involvements of anti-neutrophil cytoplasmic autoantibody-associated renal vasculitis in Japan. Nephrol Dial Transplant 30: i83-93

21. Mukhtyar C, Guillevin L, Cid MC, Dasgupta B, de Groot K, Gross W, Hauser T, Hellmich B, Jayne D, Kallenberg CG, Merkel PA, Raspe H, Salvarani C, Scott DG, Stegeman C, Watts R, Westman K, Witter J, Yazici H, Luqmani R; European Vasculitis Study Group, (2009) EULAR recommendations for the management of primary small and medium vessel vasculitis. Ann Rheum Dis 68: $310-317$

22. Levy JB, Hammad T, Coulthart A, Dougan T, Pusey CD, (2004) Clinical features and outcome of patients with both ANCA and anti-GBM antibodies. Kidney Int 66: 1535-1540

23. Cordier JF, Valeyre D, Guillevin L, Loire R, Brechot JM, (1990) Pulmonary Wegener's granulomatosis. A clinical and imaging study of 77 cases. Chest 97: 906-912

24. Frankel SK, Cosgrove GP, Fischer A, Meehan RT, Brown KK, (2006) Update in the diagnosis and management of pulmonary vasculitis. Chest 129: 452-465

25. Cottin V, Bel E, Bottero P, Dalhoff K, Humbert M, Lazor R, Sinico RA, Sivasothy P, Wechsler ME, Groh M, MarchandAdam S, Khouatra C, Wallaert B, Taille C, Delaval P, Cadranel J, Bonniaud P, Prevot G, Hirschi S, Gondouin A, Dunogue B, Chatte G, Briault A, Jayne D, Guillevin L, Cordier JF; the Groupe d'études et de recherche sur les maladies orphelines 
pulmonaires, (2016) Respiratory manifestations of eosinophilic granulomatosis with polyangiitis (Churg-Strauss). Eur Respir J 48: $1429-1441$

26. Sable-Fourtassou R, Cohen P, Mahr A, Pagnoux C, Mouthon L, Jayne D, Blockmans D, Cordier JF, Delaval P, Puechal X, Lauque D, Viallard JF, Zoulim A, Guillevin L, French Vasculitis Study G, (2005) Antineutrophil cytoplasmic antibodies and the Churg-Strauss syndrome. Ann Intern Med 143: 632-638

27. Sinico RA, Di Toma L, Maggiore U, Bottero P, Radice A, Tosoni C, Grasselli C, Pavone L, Gregorini G, Monti S, Frassi M, Vecchio F, Corace C, Venegoni E, Buzio C, (2005) Prevalence and clinical significance of antineutrophil cytoplasmic antibodies in Churg-Strauss syndrome. Arthritis Rheum 52: 2926-2935

28. Thompson G, Klecka M, Roden AC, Specks U, Cartin-Ceba R, (2016) Biopsy-proven pulmonary capillaritis: a retrospective study of aetiologies including an in-depth look at isolated pulmonary capillaritis. Respirology 21: 734-738

29. Lazor R, Bigay-Game L, Cottin V, Cadranel J, Decaux O, Fellrath JM, Cordier JF, (2007) Alveolar hemorrhage in antibasement membrane antibody disease: a series of 28 cases. Medicine 86: 181-193

30. Peto P, Salama AD, (2011) Update on antiglomerular basement membrane disease. Curr Opin Rheumatol 23: 32-37

31. Cui Z, Zhao J, Jia XY, Zhu SN, Zhao MH, (2011) Clinical features and outcomes of anti-glomerular basement membrane disease in older patients. Am J Kidney Dis 57: 575-582

32. Buret J, Marcq M, Lebranchu Y, Legras A, Barbet C, Mouteaux G, Diot P, (2008) Difficulties in the management of localised pulmonary Goodpasture's syndrome. Rev Mal Respir 25: 323-327

33. Legras A, Mordant P, Brechot N, Bel A, Boussaud V, Guillemain R, Cholley B, Gibault L, Le Pimpec-Barthes F, Combes A, (2015) Prolonged extracorporeal membrane oxygenation and lung transplantation for isolated pulmonary anti-GBM (Goodpasture) disease. Intensive Care Med 41: 1866-1868

34. Sinico RA, Radice A, Corace C, Sabadini E, Bollini B, (2006) Anti-glomerular basement membrane antibodies in the diagnosis of goodpasture syndrome: a comparison of different assays. Nephrol Dial Transplant 21: 397-401

35. Ohlsson S, Herlitz H, Lundberg S, Selga D, Molne J, Wieslander J, Segelmark M, (2014) Circulating anti-glomerular basement membrane antibodies with predominance of subclass IgG4 and false-negative immunoassay test results in anti-glomerular basement membrane disease. Am J Kidney Dis 63: 289-293

36. McAdoo SP, Tanna A, Hruskova Z, Holm L, Weiner M, Arulkumaran N, Kang A, Satrapova V, Levy J, Ohlsson S, Tesar V, Segelmark M, Pusey CD, (2017) Patients double-seropositive for ANCA and anti-GBM antibodies have varied renal survival, frequency of relapse, and outcomes compared to singleseropositive patients. Kidney Int 92: 693-702

37. Rajagopala S, Shobha V, Devaraj U, D’Souza G, Garg I, (2013) Pulmonary hemorrhage in Henoch-Schönlein purpura: case report and systematic review of the english literature. Semin Arthritis Rheum 42: 391-400

38. Martinez-Martinez MU, Oostdam DAH, Abud-Mendoza C, (2017) Diffuse alveolar hemorrhage in autoimmune diseases. Curr Rheumatol Rep 19: 27

39. Ednalino C, Yip J, Carsons SE, (2015) Systematic review of diffuse alveolar hemorrhage in systemic lupus erythematosus: focus on outcome and therapy. J Clin Rheumatol 21: 305-310

40. Retamozo S, Diaz-Lagares C, Bosch X, Bove A, Brito-Zeron P, Gomez ME, Yague J, Forns X, Cid MC, Ramos-Casals M, (2013) Life-threatening cryoglobulinemic patients with hepatitis $\mathrm{C}$ : clinical description and outcome of 279 patients. Medicine 92: $273-284$
41. Yachoui R, Sehgal R, Amlani B, Goldberg JW, (2015) Antiphospholipid antibodies-associated diffuse alveolar hemorrhage. Semin Arthritis Rheum 44: 652-657

42. Cervera R, Bucciarelli S, Plasin MA, Gomez-Puerta JA, Plaza J, Pons-Estel G, Shoenfeld Y, Ingelmo M, Espinos G; Catastrophic Antiphospholipid Syndrome Registry Project Group, (2009) Catastrophic antiphospholipid syndrome (CAPS): descriptive analysis of a series of 280 patients from the "CAPS Registry". J Autoimmun 32: 240-245

43. Cartin-Ceba R, Peikert T, Ashrani A, Keogh K, Wylam ME, Ytterberg S, Specks U, (2014) Primary antiphospholipid syndrome-associated diffuse alveolar hemorrhage. Arthritis Care Res 66: 301-310

44. Gabrilovich MI, Buxton DE, Lykins DM, McMillen SM, Onadeko OOA, (2014) Diffuse alveolar hemorrhage secondary to apixaban administration. Chest 146: e115-e116

45. Otoshi T, Kataoka Y, Nakagawa A, Otsuka K, Tomii K, (2016) Clinical features and outcomes of diffuse alveolar hemorrhage during antithrombotic therapy: a retrospective cohort study. Lung 194: 475-481

46. Moatemri Z, Zaibi H, Dabboussi S, Mhamedi S, Aichaouia C, Khadhraoui M, Cheikh R, (2016) Alveolar hemorrhage following a cannabis water pipe. Rev Pneumol Clin 72: 316-319

47. Underner M, Perriot J, Wallaert B, Peiffer G, Meurice JC, Jaafari N, (2018) Alveolar hemorrhage and cocaine use. Rev Mal Respir 35: $134-148$

48. Contou D, Voiriot G, Djibre M, Labbe V, Fartoukh M, Parrot A, (2017) Clinical features of patients with diffuse alveolar hemorrhage due to negative-pressure pulmonary edema. Lung 195: $477-487$

49. Gillet Y, Issartel B, Vanhems P, Fournet JC, Lina G, Bes M, Vandenesch F, Piemont Y, Brousse N, Floret D, Etienne J, (2002) Association between Staphylococcus aureus strains carrying gene for Panton-Valentine leukocidin and highly lethal necrotising pneumonia in young immunocompetent patients. Lancet 359: 753-759

50. Marchiori E, Lourenco S, Setubal S, Zanetti G, Gasparetto TD, Hochhegger B, (2011) Clinical and imaging manifestations of hemorrhagic pulmonary leptospirosis: a state-of-the-art review. Lung 189: 1-9

51. Niwattayakul K, Kaewtasi S, Chueasuwanchai S, Hoontrakul S, Chareonwat S, Suttinont C, Phimda K, Chierakul W, Silpasakorn S, Suputtamongkol Y, (2010) An open randomized controlled trial of desmopressin and pulse dexamethasone as adjunct therapy in patients with pulmonary involvement associated with severe leptospirosis. Clin Microbiol Infect 16: 1207-1212

52. Luks AM, Lakshminarayanan S, Hirschmann JV, (2003) Leptospirosis presenting as diffuse alveolar hemorrhage: case report and literature review. Chest 123: 639-643

53. Venkatram S, Muppuri S, Niazi M, Fuentes GD, (2010) A 24year-old pregnant patient with diffuse alveolar hemorrhage. Chest 138: 220-223

54. Ebisawa K, Yamada N, Kobayashi M, Katahira M, Konno H, Okada S, (2013) A cluster of diffuse alveolar hemorrhage cases after the 2011 Tohoku Region Pacific Coast Earthquake. Respir Investig 51: 2-8

55. Tzouvelekis A, Ntolios P, Oikonomou A, Koutsopoulos A, Sivridis E, Zacharis G, Kaltsas K, Boglou P, Mikroulis D, Bouros D, (2012) Idiopathic pulmonary hemosiderosis in adults: a case report and review of the literature. Case Rep Med 2012: 267857

56. de Prost N, Parrot A, Cuquemelle E, Picard C, Cadranel J, (2013) Immune diffuse alveolar hemorrhage: a retrospective assessment of a diagnostic scale. Lung 191: 559-563 
57. Pathak V, Kuhn J, Gabriel D, Barrow J, Jennette JC, Henke DC, (2015) Use of activated factor VII in patients with diffuse alveolar hemorrhage: a 10 years institutional experience. Lung 193: 375-379

58. Pea L, Roda L, Boussaud V, Lonjon B, (2003) Desmopressin therapy for massive hemoptysis associated with severe leptospirosis. Am J Respir Crit Care Med 167: 726-728

59. HAS, (2007) Vascularites nécrosantes. Protocole national de soins. https://www.has-sante.fr

60. Sanders JS, Rutgers A, Stegeman CA, Kallenberg CG, (2011) Pulmonary: renal syndrome with a focus on anti-GBM disease. Semin Respir Crit Care Med 32: 328-334

61. Stone JH, Merkel PA, Spiera R, Seo P, Langford CA, Hoffman GS, Kallenberg CG, St Clair EW, Turkiewicz A, Tchao NK, Webber L, Ding L, Sejismundo LP, Mieras K, Weitzenkamp D, Ikle D, Seyfert-Margolis V, Mueller M, Brunetta P, Allen NB, Fervenza FC, Geetha D, Keogh KA, Kissin EY, Monach PA, Peikert T, Stegeman C, Ytterberg SR, Specks U; Group R-IR, (2010) Rituximab versus cyclophosphamide for ANCA-associated vasculitis. N Engl J Med 363: 221-232

62. Charles P, Bienvenu B, Bonnotte B, Gobert P, Godmer P, Hachulla E, Hamidou M, Harle JR, Karras A, Lega JC, Le Quellec A, Mahr AD, Mouthon L, Papo T, Puechal X, Pugnet G, Samson M, Sibilia J, Terrier B, Vandergheynst F, Guillevin L;
FVSG, (2013) Rituximab: recommendations of the French Vasculitis Study Group (FVSG) for induction and maintenance treatments of adult, antineutrophil cytoplasm antibody-associated necrotizing vasculitides. Presse Med 42: 1317-1330

63. Yates M, Watts RA, Bajema IM, Cid MC, Crestani B, Hauser T, Hellmich B, Holle JU, Laudien M, Little MA, Luqmani RA, Mahr A, Merkel PA, Mills J, Mooney J, Segelmark M, Tesar V, Westman K, Vaglio A, Yalcindag N, Jayne DR, Mukhtyar C, (2016) EULAR/ERA-EDTA recommendations for the management of ANCA-associated vasculitis. Ann Rheum Dis 75: $1583-1594$

64. Clark WF, Huang SS, Walsh MW, Farah M, Hildebrand AM, Sontrop JM, (2016) Plasmapheresis for the treatment of kidney diseases. Kidney Int 90: 974-984

65. Cui Z, Zhao J, Jia XY, Zhu SN, Jin QZ, Cheng XY, Zhao MH, (2011) Anti-glomerular basement membrane disease: outcomes of different therapeutic regimens in a large single-center Chinese cohort study. Medicine 90: 303-311

66. Walsh M, Catapano F, Szpirt W, Thorlund K, Bruchfeld A, Guillevin L, Haubitz M, Merkel PA, Peh CA, Pusey C, Jayne D, (2011) Plasma exchange for renal vasculitis and idiopathic rapidly progressive glomerulonephritis: a meta-analysis. Am J Kidney Dis 57: 566-574 